\title{
Almost Alternating Harmonic Series
}

December 10, 2003

\section{Introduction and Examples}

One of the most important examples in the study of infinite series is the conditional convergence of the alternating harmonic series: $\sum_{n=1}^{\infty} \frac{(-1)^{n}}{n}$ converges, while $\sum_{n=1}^{\infty} \frac{1}{n}$ diverges. It seems natural, then, to ask for necessary and sufficient conditions for a sequence $\left\{a_{n}\right\}_{n=1}^{\infty}$ of 1's and -1's for which $\sum_{n=1}^{\infty} \frac{a_{n}}{n}$ converges. Consider a couple of examples:

Example 1. Let $a_{n}=-1$ if $n$ is a multiple of 3 , and $a_{n}=1$ otherwise, so that $\left\{a_{n}\right\}_{n=1}^{\infty}=\{1,1,-1,1,1,-1, \cdots\}$. Then $\sum_{n=1}^{\infty} \frac{a_{n}}{n}=\sum_{n=1}^{\infty}\left(\frac{1}{3 n-2}+\right.$ $\left.\frac{1}{3 n-1}-\frac{1}{3 n}\right)>\sum_{n=1}^{\infty} \frac{1}{3 n-2}>\sum_{n=1}^{\infty} \frac{1}{3 n}=\frac{1}{3} \sum_{n=1}^{\infty} \frac{1}{n}$, so $\sum_{n=1}^{\infty} \frac{a_{n}}{n}$ diverges.

Example 2. Let $a_{n}=1$ if $n \equiv 1$ or $2(\bmod 4)$, and $a_{n}=-1$ otherwise, so that $\left\{a_{n}\right\}_{n=1}^{\infty}=\{1,1,-1,-1,1,1,-1,-1, \cdots\}$. Then $\sum_{n=1}^{\infty} \frac{a_{n}}{n}=$ 
$\sum_{n=1}^{\infty}\left(\frac{1}{4 n-3}+\frac{1}{4 n-2}-\frac{1}{4 n-1}-\frac{1}{4 n}\right)$. Now, if we add each pair of consecutive positive terms and each pair of consecutive negative terms, we get an alternating sequence of numbers whose magnitudes approach zero monotonically. So, by the alternating series test, this series converges.

Notice that in the first example, there are, loosely speaking, twice as many 1's as -1 's, while in the second example, there are about as many 1 's as -1 's. Is this why the first example diverges while the second one converges? To make this question precise, we first need to define what we mean when we say "there are about as many 1's as -1 's." Below, we give two possible definitions. You may like one more than the other; fortunately, they are equivalent. (You might want to prove this yourself.) We will use the second definition because it turns out to be more convenient for our work here.

Definition 1. Let $\left\{a_{n}\right\}_{n=1}^{\infty}$ be a sequence of 1's and -1 's. Let $P_{n}$ denote the number of 1's and $Q_{n}$ the number of -1 's among the first $n$ terms. We say that $\left\{a_{n}\right\}_{n=1}^{\infty}$ is almost alternating if $P_{n} / Q_{n} \rightarrow 1$ as $n \rightarrow \infty$.

Definition 2. Let $\left\{a_{n}\right\}_{n=1}^{\infty}$ be a sequence of 1's and -1 's. Define the $n$th partial average of $\left\{a_{n}\right\}_{n=1}^{\infty}$ to be $A_{n}=\left(\sum_{i=1}^{n} a_{i}\right) / n$. We say that $\left\{a_{n}\right\}_{n=1}^{\infty}$ is almost alternating if $A_{n} \rightarrow 0$ as $n \rightarrow \infty$. 
Thus, the sequence in Example 1 is not almost alternating, since $A_{n} \rightarrow$ $1 / 3$ as $n \rightarrow \infty$, while the sequence in Example 2 is almost alternating, since $A_{n} \rightarrow 0$ as $n \rightarrow \infty$.

Now we can pose the informal question we asked above in more precise terms: Are the following statements true or false?

1. If $\sum_{n=1}^{\infty} \frac{a_{n}}{n}$ converges, then $\left\{a_{n}\right\}_{n=1}^{\infty}$ is almost alternating.

2. If $\left\{a_{n}\right\}_{n=1}^{\infty}$ is almost alternating, then $\sum_{n=1}^{\infty} \frac{a_{n}}{n}$ converges.

Stop at the end of this sentence and see what your intuition says about whether each of these statements is true or false! You are right if you said one is false and one is true. But which is which?

In Theorem 2 we will prove that the first statement is true. Example 4 will show that the second statement is false. Accepting for now that the first statement is true, we get another proof that the series in Example 1 diverges: $\left\{a_{n}\right\}_{n=1}^{\infty}$ is not almost alternating.

Before getting to our theorems, we consider two more examples; they can be thought of as rather extreme cases of almost alternating sequences.

Example 3. Let $\left\{a_{n}\right\}_{n=1}^{\infty}$ consist of one 1 followed by one -1 , then two 1's followed by two -1's, then three 1's followed by three -1's, etc., so 
that $\left\{a_{n}\right\}_{n=1}^{\infty}=\{1,-1,1,1,-1,-1,1,1,1,-1,-1,-1, \cdots\}$. Can you guess whether $\sum_{n=1}^{\infty} \frac{a_{n}}{n}$ converges? We'll see what the answer is shortly.

First, though, let's see that this is an almost alternating sequence. Intuitively, it seems clear that there are as many 1's as -1 's. But let's verify it carefully. Let $S_{n}=\sum_{i=1}^{n} a_{i}$, the sum of the first $n$ terms in the sequence. Observe that for $n$ a perfect square, $S_{n}=\sqrt{n}$ and thus $A_{n}=S_{n} / n=1 / \sqrt{n}$. Further, note that in fact for all $n, 0 \leq A_{n} \leq 1 / \sqrt{n}$. It follows by the Squeeze Theorem that $A_{n} \rightarrow 0$ as $n \rightarrow \infty$, so $\left\{a_{n}\right\}_{n=1}^{\infty}$ is almost alternating. Recall, though, that this by itself does not imply that the corresponding series $\sum_{n=1}^{\infty} \frac{a_{n}}{n}$ converges. We will, however, see later that this series does in fact converge.

Example 4. Define $\left\{a_{n}\right\}_{n=1}^{\infty}$ recursively as follows: Let $a_{1}=1$, and for each $n>1$, let $a_{n}=1$ unless this will cause $A_{n}>1 / \ln n$, in which case let $a_{n}=$ -1 . While we won't provide a closed form for this sequence, you may verify with a calculator that it begins as $\left\{a_{n}\right\}_{n=1}^{\infty}=\{1,1,-1,1,1,-1,1,-1, \cdots\}$ (without any "nice" pattern). Then for all $n, 0 \leq A_{n} \leq 1 / \ln n$, so again by the Squeeze Theorem, $A_{n} \rightarrow 0$ as $n \rightarrow \infty$. This time, however, the corresponding series $\sum_{n=1}^{\infty} \frac{a_{n}}{n}$ diverges, as we will see later. 


\section{Abel et al.}

It is not surprising that looking at "not-quite-alternating" sequences of 1's and -1 's is not new. In Knopp [3, p. 316], for example, we find this (Note: A null sequence is one that converges to zero.):

Given positive integers $K_{0}, K_{1}, K_{2}, \cdots$ such that $\sum(-1)^{K_{n}}$ has bounded partial sums, the series $\sum(-1)^{K_{n}} b_{n}$ converges if $\left(b_{n}\right)$ denotes any null sequence.

Actually, Knopp's statement isn't correct: to get a counterexample, let $K_{n}=$ $n$ and $b_{n}=(-1)^{n} / n$. But from what Knopp writes prior to this statement it seems that he meant to say "if $\left(b_{n}\right)$ denotes any monotone null sequence ..," which means $\left|b_{n}\right| \geq\left|b_{n+1}\right|$ for all $n$. It should be clear how this relates to our work: Suppose we have a sequence $\left\{a_{n}\right\}_{n=1}^{\infty}$ for which the partial sums are bounded. Let $b_{n}=1 / n$; then Knopp's statement (after the correction) tells us that $\sum_{n=1}^{\infty} \frac{a_{n}}{n}$ converges. This fact is a consequence of the following well-known theorem.

Theorem 1. (Abel, Dedekind, Dirichlet) Let $\left\{a_{n}\right\}_{n=1}^{\infty}$ and $\left\{b_{n}\right\}_{n=1}^{\infty}$ be sequences such that the partial sums $\sum_{n=1}^{k} a_{n}$ are bounded, $\lim _{n \rightarrow \infty} b_{n}=0$, and $\sum_{n=1}^{\infty}\left|b_{n}-b_{n+1}\right|$ converges. Then $\sum_{n=1}^{\infty} a_{n} b_{n}$ converges. 
This theorem says nothing about sequences with unbounded partial sums, such as those in Examples 3 and 4. But, surprisingly, by using the same technique that is used to prove the above theorem, we obtain theorems that do cover such examples. This technique is commonly known as summation by parts, and is due to Abel himself [1]. (Summation by parts can be thought of as a "discrete version" of integration by parts, $\int u d v=u v-\int v d u$. So, in the following, think of $x_{n}$ as $d v$ and $y_{n}$ as $u$. Then $S_{n}$ is like $v$, and $y_{n+1}-y_{n}$ is like $d u$.) We include the proof, since it is both short and relevant to our work.

Lemma 1. (Abel, 1826): Let $\left\{x_{n}\right\}_{n=1}^{\infty}$ and $\left\{y_{n}\right\}_{n=1}^{\infty}$ be sequences of real numbers, and let $S_{k}=\sum_{n=1}^{k} x_{n}$. Then

$$
\sum_{n=1}^{k} x_{n} y_{n}=S_{k} y_{k+1}+\sum_{n=1}^{k} S_{n}\left(y_{n}-y_{n+1}\right) .
$$

Proof. Note that by definition, $S_{1}=x_{1}$, and for $n>1, S_{n}-S_{n-1}=x_{n}$. Then, for any $k \geq 1$,

$$
\begin{aligned}
& \sum_{n=1}^{k} x_{n} y_{n}=x_{1} y_{1}+\sum_{n=2}^{k} x_{n} y_{n}=x_{1} y_{1}+\sum_{n=2}^{k}\left(S_{n}-S_{n-1}\right) y_{n} \\
= & S_{1} y_{1}+\sum_{n=2}^{k} S_{n} y_{n}-\sum_{n=2}^{k} S_{n-1} y_{n}=\sum_{n=1}^{k} S_{n} y_{n}-\sum_{n=2}^{k} S_{n-1} y_{n} \\
= & \sum_{n=1}^{k} S_{n} y_{n}-\sum_{n=1}^{k-1} S_{n} y_{n+1}=\sum_{n=1}^{k} S_{n} y_{n}-\sum_{n=1}^{k} S_{n} y_{n+1}+S_{k} y_{k+1}
\end{aligned}
$$




$$
=S_{k} y_{k+1}+\sum_{n=1}^{k} S_{n}\left(y_{n}-y_{n+1}\right) .
$$

\section{A Necessary Condition for Convergence}

As we said at the beginning, our goal is to find necessary and sufficient conditions on a sequence $\left\{a_{n}\right\}_{n=1}^{\infty}$ of 1's and -1 's such that $\sum_{n=1}^{\infty} \frac{a_{n}}{n}$ will converge. It turns out, however, that with almost no extra work, our analysis answers the same question in the more general case of arbitrary sequences $\left\{a_{n}\right\}_{n=1}^{\infty}$ ! Notice that although we define almost alternating only for sequences of 1's and -1 's, it is perfectly fine to define the $n$th partial average $A_{n}=\left(\sum_{i=1}^{n} a_{i}\right) / n$ for any sequence $\left\{a_{n}\right\}_{n=1}^{\infty}$ of real numbers. We can now state our first result.

Theorem 2. Let $\left\{a_{n}\right\}_{n=1}^{\infty}$ be a sequence of real numbers. If $\sum_{n=1}^{\infty} \frac{a_{n}}{n}$ converges, then $\lim _{n \rightarrow \infty} A_{n}=0$.

Proof. We write $\sum_{n=1}^{k} a_{n}$ as $\sum_{n=1}^{k} \frac{a_{n}}{n} n$, and use Abel's lemma on the latter, where $\frac{a_{n}}{n}$ plays the role of $x_{n}$, and $n$ the role of $y_{n}$. This gives $S_{k}=\sum_{n=1}^{k} \frac{a_{n}}{n}$. Thus we have

$$
\sum_{n=1}^{k} a_{n}=S_{k}(k+1)+\sum_{n=1}^{k} S_{n}(-1),
$$


so that

$$
A_{k}=\frac{1}{k} \sum_{n=1}^{k} a_{n}=S_{k} \frac{k+1}{k}-\frac{1}{k} \sum_{n=1}^{k} S_{n} .
$$

By hypothesis, $\sum_{n=1}^{\infty} \frac{a_{n}}{n}$ converges to some limit $L$. So, as $k \rightarrow \infty$, $S_{k} \rightarrow L$, and hence $S_{k} \frac{k+1}{k} \rightarrow L$. Also, $\frac{1}{k} \sum_{n=1}^{k} S_{n} \rightarrow L$, because the averages of a sequence of numbers that tend to $L$ must tend to $L$ (why?). Therefore $A_{k} \rightarrow 0$, as desired.

This theorem is similar to a theorem of Cesàro [2], which we quote from Knopp [3, p. 318]:

The relative frequency of positive and negative terms in a conditionally convergent series $\sum a_{n}$ for which $\left|a_{n}\right|$ diminishes monoton [ically] is subject to the following elegant theorem, due to E. Cesàro: The limit, if it exists, of the ratio $P_{n} / Q_{n}$ of $P_{n}$, the number of positive terms, to $Q_{n}$, the number of negative terms $a_{v}$, for $v \leq n$, is necessarily 1 .

Note that as a consequence of Theorem 2, the limit in question must indeed exist. 


\section{A Necessary and Sufficient Condition for Conver-} gence

Using Theorem 2 and another application of Abel's lemma, we have the following general result.

Theorem 3. Let $\left\{a_{n}\right\}_{n=1}^{\infty}$ be a sequence of real numbers. Then $\sum_{n=1}^{\infty} \frac{a_{n}}{n}$ converges if and only if $\lim _{n \rightarrow \infty} A_{n}=0$ and $\sum_{n=1}^{\infty} \frac{A_{n}}{n}$ converges.

Proof. In Abel's lemma, let $x_{n}=a_{n}$ and $y_{n}=1 / n$. Thus, $S_{k}=\sum_{n=1}^{k} x_{n}=$ $\sum_{n=1}^{k} a_{n}=k A_{k}$. So we get

$$
\begin{aligned}
\sum_{n=1}^{k} a_{n} \frac{1}{n} & =\sum_{n=1}^{k} x_{n} y_{n}=S_{k} y_{k+1}+\sum_{n=1}^{k} S_{n}\left(y_{n}-y_{n+1}\right) \\
& =k A_{k} \frac{1}{k+1}+\sum_{n=1}^{k} n A_{n}\left(\frac{1}{n}-\frac{1}{n+1}\right) .
\end{aligned}
$$

Rewriting $\frac{1}{n}-\frac{1}{n+1}$ as $\frac{1}{n(n+1)}$, we obtain

$$
\sum_{n=1}^{k} \frac{a_{n}}{n}=A_{k} \frac{k}{k+1}+\sum_{n=1}^{k} \frac{A_{n}}{n+1} .
$$

Now we take limits as $k \rightarrow \infty$, and use the fact that if any two of the three terms in the last equality converge, then so does the third. First though, note that $A_{k} \frac{k}{k+1} \rightarrow 0$ iff $A_{k} \rightarrow 0$; and $\sum_{n=1}^{\infty} \frac{A_{n}}{n+1}$ converges iff $\sum_{n=1}^{\infty} \frac{A_{n}}{n}$ converges (by the Limit Comparison Test). So, if $A_{k} \rightarrow 0$ and $\sum_{n=1}^{\infty} \frac{A_{n}}{n}$ 
converges, then $\sum_{n=1}^{\infty} \frac{a_{n}}{n}$ converges. Conversely, if $\sum_{n=1}^{\infty} \frac{a_{n}}{n}$ converges, then, by Theorem $2, A_{k} \rightarrow 0$, and hence $\sum_{n=1}^{\infty} \frac{A_{n}}{n}$ converges, as desired.

We now look at how this theorem applies to the series in Examples 3 and 4 . Recall that in both cases, we have already established that $A_{n} \rightarrow 0$. Thus, in each case we must check whether or not $\sum_{n=1}^{\infty} \frac{A_{n}}{n}$ converges.

Example 3. (Continued) We saw that for all $n, 0 \leq A_{n} \leq 1 / \sqrt{n}$, so that $0 \leq A_{n} / n \leq 1 / n^{3 / 2}$. But $\sum_{n=1}^{\infty} \frac{1}{n^{3 / 2}}$ is a convergent $p$-series, so $\sum_{n=1}^{\infty} \frac{A_{n}}{n}$ converges by comparison. Therefore by Theorem $3, \sum_{n=1}^{\infty} \frac{a_{n}}{n}$ converges.

Example 4. (Continued) Recall that $\left\{a_{n}\right\}_{n=1}^{\infty}$ is defined recursively: $a_{1}=1$, and for $n \geq 2$,

$$
a_{n}=\left\{\begin{array}{ll}
1 & \text { if } \frac{\left(\sum_{i=1}^{n-1} a_{i}\right)+1}{n} \leq \frac{1}{\ln n} \\
-1 & \text { otherwise }
\end{array} .\right.
$$

The purpose of this seemingly strange way of defining $\left\{a_{n}\right\}_{n=1}^{\infty}$ is precisely so that its $n$th partial average $A_{n}$ stays "close" to $1 / \ln n$. Then we can expect that, loosely speaking, $\sum_{n=1}^{\infty} \frac{A_{n}}{n} \approx \sum_{n=1}^{\infty} \frac{1}{n \ln n}$, which is known to diverge by the integral test.

A rigorous proof, however, takes a bit of work, but is a good exercise in 
mathematical induction: we prove that $\forall n \geq 2$,

$$
A_{n}>\frac{1}{\ln n}-\frac{2}{n}
$$

Base Step: Since $a_{1}=a_{2}=1, A_{2}=1>1 / \ln 2-2 / 2$.

Induction Step: Assume (1) holds for some $n=k$. By definition,

$$
A_{n+1}=\frac{a_{1}+\cdots a_{n+1}}{n+1}=\frac{n A_{n}+a_{n+1}}{n+1} .
$$

We consider two cases.

Case 1: $a_{k+1}=-1$. Then, by the definition of $\left\{a_{n}\right\}_{n=1}^{\infty}$,

$$
\frac{n A_{n}+1}{n+1}>\frac{1}{\ln (n+1)}
$$

Subtracting $2 /(n+1)$ from both sides gives

$$
\frac{n A_{n}-1}{n+1}>\frac{1}{\ln (n+1)}-\frac{2}{n+1}
$$

But the left-hand side is exactly $A_{n+1}$, so we're done with this case.

Case 2: $a_{k+1}=1$. Then, combining Equation 2 with the induction hypothesis, i.e. inequality (1), we get

$$
A_{n+1}>\frac{n\left(\frac{1}{\ln n}-\frac{2}{n}\right)+1}{n+1}=\frac{n}{(n+1) \ln n}-\frac{1}{n+1} .
$$

Thus, to prove the induction step for $A_{n+1}$, it is enough to show that for $n \geq 2$,

$$
\frac{n}{(n+1) \ln n}-\frac{1}{n+1} \geq \frac{1}{\ln (n+1)}-\frac{2}{n+1}
$$


which is equivalent to

$$
\frac{n}{\ln n}+1 \geq \frac{n+1}{\ln (n+1)}
$$

This last inequality holds because for the function $f(x)=x / \ln x, f^{\prime}(x)<1$ for $x \geq 2$, and hence $f(x)+1>f(x+1)$.

Thus, the series $\sum_{n=1}^{\infty} \frac{a_{n}}{n}$ does not converge.

It should be noted that Example 4 shows that the converse to Theorem 2 does not hold.

\section{A More Specific Equivalence}

Recall that in Theorem $3,\left\{a_{n}\right\}_{n=1}^{\infty}$ is an arbitrary sequence of real numbers. In this section, we show that the condition $\lim _{n \rightarrow \infty} A_{n}=0$ of that theorem is actually redundant if $\left\{a_{n}\right\}_{n=1}^{\infty}$ is a sequence of 1's and -1's. We will need a somewhat technical lemma.

Lemma 2. Let $\left\{x_{n}\right\}_{n=1}^{\infty}$ be a sequence of real numbers such that $n\left|x_{n+1}-x_{n}\right|$ is bounded above. Then $\forall \epsilon>0, \exists \delta>0$ such that for all $i, k$ with $i<k$, if $\left|x_{i}\right| \leq \epsilon$ and $\left|x_{k}\right| \geq 2 \epsilon$, then $\left|\sum_{n=j}^{k} \frac{x_{n}}{n}\right|>\delta$ for some $j \in\{i, \cdots, k\}$.

Proof. Let $\epsilon>0$ and $i<k$ be given, with $\left|x_{i}\right| \leq \epsilon$ and $\left|x_{k}\right| \geq 2 \epsilon$. We want to find a $\delta$ that depends on $\epsilon$ but not on $i$ and $k$, and satisfies the conclusion 
of the lemma. Without loss of generality, we can assume $x_{k} \geq 2 \epsilon$, since otherwise we could instead work with the sequence $\left\{y_{n}\right\}_{n=1}^{\infty}=\left\{-x_{n}\right\}_{n=1}^{\infty}$ to find the same $\delta$. Pick the smallest $j \in\{i, \cdots, k-1\}$ such that $\forall n \in$ $\{j+1, \cdots, k\}, x_{n} \geq \epsilon$. Then

$$
\sum_{n=j+1}^{k} \frac{x_{n}}{n} \geq \sum_{n=j+1}^{k} \frac{\epsilon}{n} \geq \sum_{n=j+1}^{k} \frac{\epsilon}{k}=(k-j) \frac{\epsilon}{k} .
$$

We'd like to find a lower bound for $(k-j) / k$; we'll first find one for $(k-j) / j$. By hypothesis, $\forall n \in\{j, \cdots, k-1\}$, each difference $x_{n+1}-x_{n}$ has magnitude less than $\alpha / n \leq \alpha / j$, where $\alpha$ is an upper bound for $n\left|x_{n+1}-x_{n}\right|$. From $n=j$ to $n=k-1$ we have $k-j$ such differences, which together add up to $x_{k}-x_{j} \geq \epsilon$, since $x_{k} \geq 2 \epsilon$ and $x_{j} \leq \epsilon$. So $(k-j) \alpha / j>\epsilon$, which gives

$$
\frac{k-j}{j}>\frac{\epsilon}{\alpha}
$$

Now, to get a lower bound for $(k-j) / k$, we consider two cases:

Case 1: $k \leq 2 j$. Then $\frac{k-j}{k} \geq \frac{k-j}{2 j}>\frac{\epsilon}{2 \alpha}$.

Case $2: k>2 j$. Then $k-2 j>0$, so $2 k-2 j>k$, so $\frac{k-j}{k}>\frac{1}{2}$.

In either case, $\frac{k-j}{k}>\min \left(\frac{1}{2}, \frac{\epsilon}{2 \alpha}\right)$. So if we let $\delta=\min \left(\frac{\epsilon}{2}, \frac{\epsilon^{2}}{2 \alpha}\right)$, then inequality 3 gives us $\sum_{n=j+1}^{k} \frac{x_{n}}{n} \geq \delta$, as desired.

Lemma 3. Let $\left\{x_{n}\right\}_{n=1}^{\infty}$ be a sequence of real numbers such that $n\left|x_{n+1}-x_{n}\right|$ is bounded above. If $\sum_{n=1}^{\infty} x_{n} / n$ converges, then $\lim _{n \rightarrow \infty} x_{n}=0$. 
Proof. Suppose, towards contradiction, that $\lim _{n \rightarrow \infty} x_{n} \neq 0$. Then $\exists \epsilon>0$ such that $\left|x_{n}\right|>2 \epsilon$ for infinitely many $n$; that is, $\forall N, \exists n>N$ such that $\left|x_{n}\right|>2 \epsilon$.

We also claim that then $\left|x_{n}\right| \leq \epsilon$ for infinitely many $n$. For suppose not; that is, $\exists M$ such that $\forall n>M,\left|x_{n}\right|>\epsilon$. Let $\alpha$ be an upper bound for $n\left|x_{n+1}-x_{n}\right|$. Then for $n>\alpha / \epsilon,\left|x_{n+1}-x_{n}\right|<\alpha / n<\epsilon$. Therefore, either $x_{n}>\epsilon$ for all $n>\max (M, \alpha / \epsilon)$, or $x_{n}<-\epsilon$ for all $n>\max (M, \alpha / \epsilon)$. But then comparison with the harmonic series would prove that $\sum_{n=1}^{\infty} \frac{x_{n}}{n}$ diverges, which contradicts the hypothesis.

Thus $\left|x_{n}\right| \leq \epsilon$ for infinitely many $n$ and $\left|x_{n}\right| \geq 2 \epsilon$ for infinitely many $n$. Then, by the previous lemma, $\exists \delta>0$ such that for infinitely many pairs $j, k$ we have $\left|\sum_{n=j}^{k} \frac{x_{n}}{n}\right|>\delta$. This contradicts the hypothesis that $\sum_{n=1}^{\infty} \frac{x_{n}}{n}$ converges.

We invite the reader to find a sequence $\left\{a_{n}\right\}_{n=1}^{\infty}$ of real numbers for which the following lemma and theorem do not hold.

Lemma 4. Let $\left\{a_{n}\right\}_{n=1}^{\infty}$ be a sequence of 1 's and -1 's. If $\sum_{n=1}^{\infty} \frac{A_{n}}{n}$ converges, then $\lim _{n \rightarrow \infty} A_{n}=0$. 
Proof. This follows immediately from Lemma 3 and the fact that for all $n$, $\left|A_{n+1}-A_{n}\right|<2 / n$, which is seen as follows (note that for all $n,\left|A_{n}\right| \leq 1$ since $\left\{a_{n}\right\}_{n=1}^{\infty}$ is a sequence of 1's and -1 's):

$$
\begin{aligned}
& \left|A_{n+1}-A_{n}\right|=\left|\frac{n A_{n} \pm 1}{n+1}-A_{n}\right|=\left|\frac{n A_{n} \pm 1-(n+1) A_{n}}{n+1}\right| \\
& =\frac{1}{n+1}\left| \pm 1-A_{n}\right| \leq \frac{1}{n+1}\left(1+\left|A_{n}\right|\right) \leq \frac{1}{n+1}(1+1)<\frac{2}{n} .
\end{aligned}
$$

Finally, then, we obtain the following necessary and sufficient condition by combining Theorem 3 and Lemma 4 .

Theorem 4. Let $\left\{a_{n}\right\}_{n=1}^{\infty}$ be a sequence of 1 's and -1 's. Then $\sum_{n=1}^{\infty} \frac{a_{n}}{n}$ converges if and only if $\sum_{n=1}^{\infty} \frac{A_{n}}{n}$ converges.

\section{Acknowledgments:}

We would like to thank Masato Kimura of Hood College for the idea of studying sequences of 1's and -1's and looking at $n$th partial averages. We also thank Wilhelm Schlag of Caltech for showing us a proof of Theorem 2 which is much simpler than our original (and which we were able to further simplify into its current form). The second author is grateful to Caltech for its hospitality while he worked on this article during part of his sabbatical leave. 


\section{References}

[1] Neils Henrik Abel, J. Reine Angew. Math. 1 (1826), 314.

[2] E. Cesàro, Rom. Acc. Lincei Rend. 4 (1888), 133.

[3] Konrad Knopp, Theory and application of infinite series (Trans. R. C. H. Young), Hafner, 1947.

Curtis Feist: Mathematics Department, Southern Oregon University, Ashland, OR 97520, USA. feistc@sou.edu.

Ramin Naimi: Mathematics Department, Occidental College, Los Angeles, CA 90041, USA. rnaimi@oxy.edu. 